\title{
Neurólise ulnar sob anestesia local em pacientes com hanseníase
}

\author{
José Marcos Pondét, Marcos Antônio Falcão da Silva², \\ Icaro Barros Barreto ${ }^{3}$, Clarissa Cerqueira Ramos ${ }^{3}$ \\ Hospital Colônia Dom Rodrigo de Menezes da Secretaria de Saúde do Estado da Bahia, Salvador, BA.
}

\section{RESUMO}

Contexto: A hanseníase é uma doença endêmica em nosso meio e pode provocar deformidades físicas se não tratada adequadamente. A descompressão cirúrgica do nervo periférico acometido por vezes é necessária para prevenção dessas ocorrências. Objetivo: Relatar a técnica de descompressão de nervo ulnar, o mais frequentemente acometido na hanseníase, realizada ambulatorialmente em serviço de referência no estado da Bahia. Material e métodos: Os autores relatam a experiência com 84 pacientes submetidos à cirurgia ambulatorial de neuropatia compressiva ulnar, entre fevereiro de 2008 e fevereiro de 2009. O quadro clínico mais encontrado foi dor de difícil manejo terapêutico e as incapacidades físicas. As cirurgias tiveram uma média de duração de 30 minutos. O procedimento consistiu em descompressão seguida de neurólise de nervo ulnar sob anestesia local sem utilização de garrote. Resultados: Os pacientes toleraram bem as cirurgias, e a dor, avaliada pela escala analógica, melhorou em praticamente todos os casos. Conclusão: A técnica ambulatorial de descompressão ulnar é um método eficaz, com baixo índice de complicações e adequada para manuseio de doença tão desafiadora como a hanseníase em países em desenvolvimento.

\section{PALAVRAS-CHAVE}

Neurólise de ulnar. Hanseníase.

\begin{abstract}
Ulnar nerve decompression with local anesthesia in patients with Hansen disease

Background: Leprosy is endemic in our country leading often to physical deformities when not adequately treated. The surgical decompression of the peripheral nerve involved by the disease sometimes is the best option in order to avoid these occurrences. Objective: To report the surgical technique of ulnar decompression with local anesthesia as an outpatient procedure at a Leprosy Institution in Bahia, Brazil. Material and methods: Eighty-four outpatients were operated between February 2008 and February 2009. Pain of difficult management and the physical disabilities were the predominant symptoms. The procedure consisted of decompression followed by neurolyses under local anesthesia without tourniquet. The median surgical time was thirty minutes. Results: The patients tolerated well the procedure and significant pain relief was obtained in almost all cases. Conclusion: The outpatient surgical decompression of ulnar nerve is efficacious and accompanied by minor complications and is adequate to manage ulnar compression by leprosy in developing countries.
\end{abstract}

\section{KEY WORDS}

Ulnar neurolysis. Leprosy.

\section{Introdução}

A lepra foi identificada em civilizações remotas como a China, o Egito e a Índia. O primeiro relato data de 600 a.C. Em 1864, Gerhard Armour Hansen correlacionou em um paciente na Noruega o bacilo com a afecção.
A doença tem baixa infectividade, com tempo de incubação médio de cinco anos, e a transmissão ocorre por contato cutâneo e por via aérea. ${ }^{1}$ Existem aproximadamente 10 milhões de pessoas acometidas pela doença no mundo, a maior parte em países em desenvolvimento da África, Ásia e América Latina, apesar de 95\% dos indivíduos terem imunidade natural à doença.

1. Professor adjunto doutor da Faculdade de Medicina, Departamento de Neurociências e Saúde Mental, da Universidade Federal da Bahia (UFBA), Salvador, BA.

2. Fisioterapeuta do Hospital Dom Rodrigo de Menezes, Secretaria de Saúde do Estado da Bahia, Salvador, BA.

3. Aluno(a) de graduação da Escola Bahiana de Medicina e Saúde Pública (EBMSP), Salvador, BA. 
A lepra é uma doença de caráter endêmico em nosso meio. O Brasil, em números absolutos, possui a segunda população mundial com essa doença, ficando atrás apenas da Índia. Porém, em números relativos supera todos os demais países onde a doença aparece de forma endêmica.

O envolvimento do sistema nervoso periférico é comum na lepra e resulta em danos que levam a diversas deformidades. ${ }^{3-5,12} \mathrm{Na}$ forma dita multibacilar, o espessamento do nervo ocorre por invasão direta do bacilo no tecido neural ou devido às reações, enquanto na forma paucibacilar as reações de hipersensibilidade levam a inflamação súbita e edema neural, conduzindo ao quadro de síndromes compartimentais diversas. Frequentemente, o parênquima neural é substituído por tecido fibroso. ${ }^{8}$ Os sítios de predileção do bacilo, usualmente, são locais de temperatura corporal mais baixa, em torno de 27 a 30 graus Celsius. ${ }^{1,5,6}$

A perda de sensibilidade é o sintoma mais relatado, seguida por déficit motor. As alterações autonômicas levam ao ressecamento da pele e à formação de fissuras. Os déficits motores acarretam a mão em garra e o pé caído, e as alterações sensitivas conduzem a úlceras tróficas. $^{5-7}$

Ao tratamento medicamentoso específico se associa, frequentemente, o uso de corticoides que visam reduzir os processos reacionais e melhorar a dor. ${ }^{8,11}$

A cirurgia é reservada para os casos em que não existe melhora dos sintomas após quatro meses de tratamento clínico, na presença de abscesso do nervo ou, precocemente, nos casos de envolvimento grave do sistema nervoso periférico sem resposta clínica.

\section{Objetivo}

Mostrar a técnica utilizada em 95 cirurgias de neurólise de nervo ulnar realizadas em 84 pacientes portadores de neuropatia compressiva no curso da moléstia de Hansen.

\section{Casuística e método}

Foram operados 135 pacientes com neuropatia compressiva, totalizando 286 cirurgias, entre fevereiro de 2008 e fevereiro de 2009, no Hospital Colônia Dom Rodrigo de Menezes da Secretaria de Saúde do Estado da Bahia. A média de idade foi de 41,06 anos, com desvio-padrão de 14,57, variando de 6 a 87 anos; 93 pacientes eram do sexo masculino e 42, do feminino;
84 foram selecionados por serem portadores de compressão ulnar no curso de tratamento da hanseníase.

Os pacientes tinham diagnóstico confirmado por meio de exame baciloscópico ou biópsia. A média entre o aparecimento dos sintomas e o diagnóstico foi de 11 meses, sendo o mais precoce um mês e o mais tardio 50 meses.

Os pacientes foram avaliados por um único fisioterapeuta no pré-operatório e três e seis meses após o procedimento cirúrgico. A avaliação consistiu no teste de sensibilidade utilizando filamentos de nylon segundo o método de Siemmens Weinberg, avaliação da força muscular e do grau de incapacidade com escalas específicas.

A sensibilidade foi quantificada numericamente de 0 a 5 nos dermátomos correspondentes ao nervos ulnar e mediano no membro superior. $\mathrm{O}$ valor 0 corresponde à anestesia total e o valor 5 , à sensibilidade normal, com os restantes valores intermediários sendo quantificados de acordo com o paciente.

A dor foi quantificada por meio de escala analógica visual com valores de 0 a 10 .

Nos 84 portadores de compressão ulnar, foi realizado um total de 95 cirurgias.

Tanto o lado direito quanto o esquerdo foram acometidos em frequências similares: 45 no direito e 50 no esquerdo; destes, 11 foram operados bilateralmente $(12 \%)$.

\section{Técnica cirúrgica}

Paciente em decúbito dorsal horizontal com membro superior abduzido e semiflexão do cotovelo. Antissepsia com polvidine alcoólico. Bloqueio anestésico do nervo no cotovelo, $2 \mathrm{~cm}$ acima do sulco do nervo ulnar, com $5 \mathrm{ml}$ de xylocaína a $2 \%$ com vasoconstrictor (Figura 1). Anestesia do subcutâneo com $15 \mathrm{ml}$ de xylocaína a $2 \%$ na face anteromedial do cotovelo no local da incisão cirúrgica. Incisão curvilínea na face anteromedial do cotovelo com cerca de $5 \mathrm{~cm}$ de extensão. Hemostasia dos vasos sangrantes com bipolar. Dissecção do plano subcutâneo penetrando na fáscia superficial, de sorte a deixar a fáscia profunda subcutânea para ser utilizada posteriormente, aderida aos músculos. Visualização dos músculos inseridos no epicôndilo medial (pronador redondo, palmar longo, flexor ulnar do carpo, flexor superficial e profundo dos dedos). Liberação do nervo ulnar do seu canal entre os epicôndilos medial e lateral. O nervo ulnar, proximalmente, é liberado até alcançar a arcada de Struthers quase sempre presente. Distalmente, liberação por meio da secção entre os dois feixes do flexor ulnar do carpo (Figura 2). Os ramos articulares do nervo ulnar são seccionados para sua completa liberação. Secção 
do epineuro com liberação do nervo nos sentidos proximal e distal, tendo como reparo para o final da dissecção o ramo motor para o músculo flexor ulnar do carpo, que é mantido intacto. Nessa etapa, faz-se o descolamento da fáscia subcutânea profunda dos seus músculos subjacentes e esse tecido é utilizado para proteção do nervo ventralizado. Fechamento do canal músculo-aponeurótico com pontos separados de fio mononylon 2-0. Ventralização do nervo deitando sobre os músculos do epicôndilo medial e protegido com a fáscia subcutânea anteriormente descolada e fixada com fio Vycril 2-0. Fechamento em dois planos com fio Vycril 2-0 no subcutâneo e mononylon 3-0 em ponto contínuo simples na pele. Curativo oclusivo sem compressão.

A média de tempo cirúrgico foi de 30 minutos. Os pacientes foram operados em regime ambulatorial com retirada de pontos cirúrgicos em três semanas.

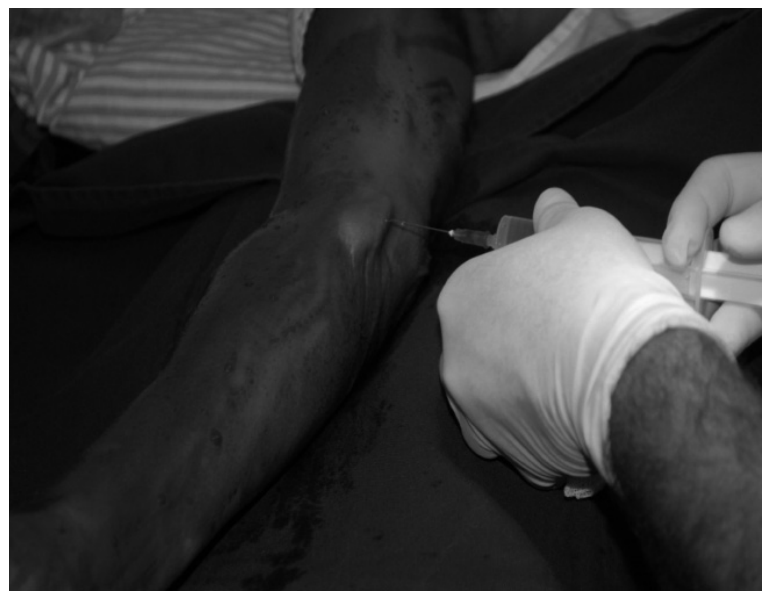

Figura 1 - Bloqueio anestésico.

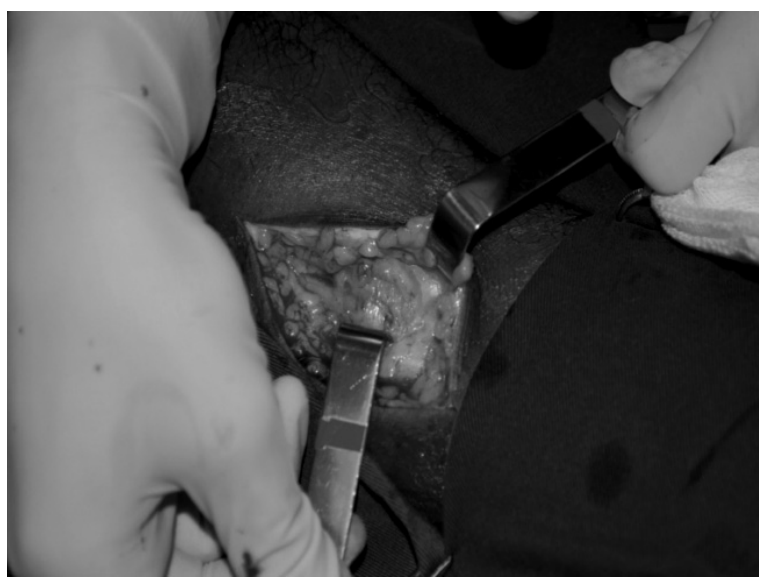

Figura 2 - Compressão no sulco do nervo ulnar.

\section{Resultados}

Os pacientes toleraram os procedimentos de forma bastante satisfatória, sem necessidade de complementação da dose anestésica, sendo apenas referida leve disestesia durante a manipulação do nervo no instante da ventralização na maior parte dos pacientes.

Os pacientes, na sua imensa maioria, referiram melhora importante do quadro doloroso no pós-operatório, e a análise estatística com o teste T de Student mostrou valores significativos $(p<0,05)$.

A única complicação, observada em poucos casos e sem consequência, foi a deiscência de sutura.

\section{Discussão}

A doença acomete o sistema cutâneo e os nervos periféricos, tendo predileção por áreas mais frias do corpo como a face e as extremidades. ${ }^{1,5,6,12}$

A disposição anatômica de alguns nervos atravessa canais naturais; estes acabam funcionando como pontos de angustiamento desses nervos hipertrofiados em decorrência da doença. ${ }^{5-7}$ Vale mencionar o fato de que nos membros superiores comumente o lado esquerdo é o mais acometido, por ser o normalmente menos exercitado no dia a dia e consequentemente menos aquecido.

Os locais mais acometidos são o cotovelo, o canal carpiano, o túnel do tarso e a cabeça da fíbula, comprometendo respectivamente os nervos ulnar, mediano, tibial posterior e fibular. ${ }^{5-7}$

A descompressão cirúrgica seguida de neurólise é defendida por diversos autores como forma de prevenir as deformidades como mão em garra, mal perfurante plantar, e assim por diante. ${ }^{4,5,8,9}$

Fato comum em todas essas situações clínicas é um incremento volumétrico no conteúdo dos canais naturais, sem um correspondente aumento do continente, o que gera um mecanismo de lesão secundária, muitas vezes mais significativo que a própria doença.

Essa peculiaridade da hanseníase é oposta à encontrada na neuropatia compressiva clássica, habitualmente vista no membro superior direito.

A indicação cirúrgica se baseou nos critérios clínicos, levando em consideração a escala de sensibilidade com os fios de Siemmens Weinberg, a perda de força muscular e a presença de alterações tróficas. A sensibilidade variou de 0 a 5 , sendo o nível 3 a chamada sensibilidade protetora, nível abaixo do qual os doentes com hanseníase sofrem lesões cutâneas que passam despercebidas. 


\section{Conclusão}

O método utilizado de anestesia local ambulatorial para descompressão de nervo ulnar se constitui numa técnica segura e eficaz no manuseio de enfermidade tão prevalente em nosso meio.

\section{Referências}

1. Agrawal A, Pandit L, Dalal M, Shetty JP. Neurological manifestations of Hansen's disease and their management. Clin Neurol Neurosurg. 2005;107:445-54.

2. Bryceson A, Pfaltzgraff RE. Medicine in the tropics: leprosy. $3^{\text {rd }}$ ed. Edinburgh: Churchill Livingstone; 1990. p. 133-51.

3. Croft RP, Nicholls PG, Steyerberg EW, Richardus JH, Cairns W, Smith S. A clinical prediction rule for nervefunction impairment in leprosy patients. Lancet. 2000;355:1603-6.

4. Girdhar Bk. Neuritic leprosy. Ind J Lepr. 1996;68:35-42.

5. Husain S, Mishra B, Malaviya GN. Evaluation of results of surgical decompression of median nerve in leprosy in relation to sensory motor function. Acta Leprol. 1997;10:199-201.
6. Husain S, Mishra B, Malaviya GN. Nerve trunk decompression in lower limb in leprosy. Indian J Orthop. 1998;4:85-7.

7. Husain S, Mishra B, Prakash V, Malaviya GN. Results of surgical decompression of ulnar nerve in leprosy. Acta Leprol. 1998;11:17-20.

8. Naafs B. Bangkok Workshop on Leprosy Research. Treatment of reactions and nerve damage. Int J Lepr Other Mycobact Dis. 1996;64(4 Suppl.):S21-8.

9. Pandya NJ. Surgical decompression of nerves in leprosy: an attempt at prevention of deformities: a clinical, electrophysiologic, histophatologic and surgical study. Int J Lepr Other Mycobact Dis. 1978;46:47-55.

10. Ridley MJ, Waters MFR, Ridley DS. Effect of mycobacterium leprae in the peripheral nerve trunk on the evolution of skin lesions. Int J Lepr. 1994;62:99-107.

11. Sugumaran DS. Steroid therapy for paralytic deformities in leprosy. Int J Lepr Other Mycobact Dis. 1997;65:337-44.

12. Van Brakel WH. Peripheral neuropathy in leprosy and its consequences. Lepr Rev. 2000;71(Suppl):S 146-53.

Endereço para correspondência

Icaro Barros Barreto

Avenida Paulo VI, 111

41810-000 - Pituba, BA

E-mail: icarobarros180@hotmail.com 